\title{
Classification in Terms of Basic Concepts
}

\author{
ASIS\&T SIG-CR Workshop 2012 \\ Rick Szostak \\ University of Alberta \\ rszostak@ualberta.ca
}

\section{KEYWORDS}

Basic Concepts, Interdisciplinarity, Subject Headings

\section{EXTENDED ABSTRACT}

arguments:

My presentation follows a set of logical

1. The vast majority of scholarly research addresses how one or more things affect in some way(s)

one or more other things. This is true of much of the general literature too:

(gardener)(grow)(flowers)

(dogs)(bite)(mail carriers)

[I illustrate this point in Szostak (2003). Note

that works that describe only one thing will also

be well handled by the classification outlined

below.]

2. If a book is about dogs biting mail carriers, the ideal subject entry is "(mail carriers)(bitten by)(dogs)." The classification system then reflects the actual structure of works.

3. This is best done by allowing 'things' (dogs, mail carriers) and 'relationships' (biting) to be freely combined in both classification and search (In the right order, so as not to mistakenly recall the smaller but more intriguing literature on mail carriers biting dogs.). This spares the classificationist from having to enumerate a vast array of combinations, and the user from having to ascertain how a particular combination was treated.

4. The real beauty of this approach is that the terms "dog," "biting," and "mail carrier" are far less ambiguous than any term the classificationist might have derived to capture this complex relationship. (Szostak, 2011).

5. So we simultaneously get:

- Subject headings that better capture the essence of works

Advances in Classification Research 2012, October 26, 2012, Baltimore, MD, USA.
- Subject headings that are less ambiguous

- Far shorter schedules, because the vast majority of existing subject headings capture (often poorly) combinations among a much smaller set of things and relationships

- Greater flexibility, for new subjects will generally be found to be combinations of things and relationships that are already in the schedules (The need for greater flexibility in classification was stressed by Grant Campbell in his presentation)

- Superior hierarchical structures. At present, many classifications squeeze causal statements into hierarchical structures of subjects. In the proposed classification, hierarchical subdivision could proceed according to logical principles, since causal arguments will be handled through combinations of terms (Dave Durbin discussed challenges in hierarchy during his presentation).

6. This approach was extremely difficult with card catalogues but is easy now.

7. It is an empirical question as to whether this approach supports a truly universal classification. (We should not assume this result. The philosophical literature establishes that some degree of ambiguity is inevitable but cannot by its nature determine how much) (Again see Szostak 2011)

8. This approach reduces ambiguity as much as it is possible to do. That is, the ambiguity-minimizing strategy is to break complex concepts into their more basic components.

9. Only by developing a wide-ranging classification of things and relationships, and showing that these can be combined to (better) capture the essence of existing subject headings (or classes), can we then answer the empirical question. 
Szostak, R. (2013). Classification in Terms of Basic Concepts. Advances In Classification Research Online, 23(1), pp. 33-34. doi:10.7152/acro.v23i1.14234

10. This has been done:

The Basic Concepts Classification http://www.economics.ualberta.ca/en/Facultyand Staff/ /media/economics/FacultyAndStaff/Szost ak/Szostak-Basic-Concept-Classification2.pdf

And my translation of 300-340 in DDC into BCC (which often increased clarity, and only rarely required more than 3-4 basic terms). http://www.economics.ualberta.ca/en/Facultyand Staff/ /media/economics/FacultyAndStaff/Szost ak/Szostak-Dewey-Conversion-Table.pdf

(See also the Integrative Levels Classification at www.iskoi.org/ilc)

11. We need to exercise collective judgment as to whether this approach renders a truly universal classification feasible. It deserves to be stressed again that we should not assume any particular degree of ambiguity at the outset.

12. A positive result is important because such a classification:

- Will greatly facilitate interdisciplinary research.

- Yet at the same time allows disciplinary (specialized) research to continue as it has, but the new classification will both facilitate within-discipline searches and increase the likelihood that specialized researchers will become aware of related research in other fields.

13. These results are even truer if time allowed discussion of the other critical element of BCC: classifying works also in terms of theory and method applied (see Szostak 2004, Gnoli and Szostak 2008).

14. Indeed, I have become convinced that the most important key to unlocking the potential of interdisciplinarity lies in the development of better classification systems (For two decades my research has focused on how to best facilitate interdisciplinary research (and teaching)

15. The proposed system:

- Allows users to better find what they are looking for in any field (since they can search by combinations of things, relationships, theories, methods, and perspectives applied).

- Dramatically increases the chance of finding related work that one didn't know to look for (since works from other perspectives addressing the same or similar questions will be easily found).

- Increases our ability to understand works from other fields once we locate them (since the practice of breaking complex concepts into more basic concepts facilitates understanding).

16. The same applies to any groups: The proposed classification will enhance the possibility of cross-group understanding, while also facilitating within-group conversation. (Szostak 2012)

17. Moreover, a classification grounded in basic concepts may well be the only way of achieving the goal of having multiple databases utilizing the same classification. Commercial and even non-profit websites are unlikely to adopt an existing KOS but may adopt one that allows combinations of simple terms:

(shoes)(for)(hiking)(desert)

Going forward, my research agenda involves:

- Continuing to Develop BCC (I am working in particular on natural science and humanities)

- User testing

- Continuing to argue for both the desirability and feasibility of the system (I plan on looking at samples from LCSH, especially 'new additions', and showing how these are generally combinations of simpler terms contained in the BCC) [I am also co-authoring a book on "Interdisciplinary Knowledge Organization"]

I invite:

- Comments

- Critiques

but especially

- Collaboration. I have benefitted enormously from my previous interactions with scholars such as Claudio Gnoli, and invite others to join in the development of the first truly universal classification. This is a moment in time - as new online websites proliferate - when such a project has a considerable chance of success.

\section{REFERENCES}

Gnoli, C. \& Szostak, R. (2008) Classifying by phenomena, theories, and methods: examples with focused social science theories, in Culture and identity in knowledge organization, proceedings of the 10th international ISKO conference, Montréal, 2008 (pp. 205-211) Würzburg: Ergon.

Szostak, R. (2012) Speaking Truth to Power in Classification. Letter to the editor. Forthcoming, Knowledge Organization 40:1.

Szostak, R. (2011). "Complex Concepts into Basic Concepts" Journal of the American Society for Information Science \& Technology. 62:11, 2247-65

Szostak, R. (2004) Classifying Science: Phenomena, Data, Theory, Method, Practice, Dordrecht: Springer.

Szostak, R. (2003) A Schema for Unifying Human Science: Interdisciplinary Perspectives on Culture, Selinsgrove PA: Susquehanna University Press. 